1. Ezard, T. H. G., Aze, T., Pearson, P. N. \& Purvis, A. Science 332, 349-351 (2011).

2. Żliobaitè, I., Fortelius, M. \& Stenseth, N. C. Nature 552, 92-95 (2017)

3. Fortelius, M. et al. Phil. Trans. R. Soc. B 371 , 20150232 (2016)

4. Žliobaitè, I. et al. Proc. Natl Acad. Sci. USA 113, 12751-12756 (2016)
5. Van Valen, L. Evol. Theory 1, 1-30 (1973).

6. Quental, T. B. \& Marshall, C. R. Science 341, 290-292 (2013)

7. Van Valen, L. Evol. Theory 1, 179-229 (1976).

8. Foote, M. et al. Science 318, 1131-1134 (2007).

9. Sepkoski, J. J. Jr Paleobiology 7, 36-53 (1981)

10.Jablonski, D. Evol. Biol. 44, 427-450 (2017).
11.Alroy, J. Quantitative Mammalian Biochronology and Biogeography of North America. PhD thesis (1994).

12. Foote, M. \& Miller, A. I. Principles of Paleontology 185 (Fig. 7.11) (Freeman, 2006)

13. Marshall, C. R. Nature Ecol. Evol. 1, 0165 (2017).

14.Gould, S. J. Paleobiology 6, 96-118 (1980)

This article was published online on 29 November 2017.

\title{
A steamy proposal for Martian clays
}

Martian clays present a conundrum: the models proposed to explain their formation require conditions that are not predicted by computational climate simulations. Experiments now suggest an alternative scenario. SEE LETTER P.88

\section{LAURA SCHAEFER}

$\longrightarrow$ lay minerals are found in abundance across the surface of Mars, and many models have been suggested to explain their formation ${ }^{1}$. These models often invoke the presence of substantial surface water and a warm climate during Mars's first 500 million to 700 million years. However, computational climate models for early Mars struggle to reproduce such conditions ${ }^{2}$. On page 88 , Cannon et al. ${ }^{3}$ present a possible solution to this problem: a model for clay formation during the end stages of Mars's formation that does not require prolonged warm and wet conditions.

During their formation, many planets go through a stage known as a magma ocean, which results from substantial or complete melting of their interiors. Both Earth and Mars went through at least one such phase ${ }^{4}$. The melting of their silicate mantles led to outgassing of volatile components that were originally incorporated in the solid material. This produced atmospheres containing one or
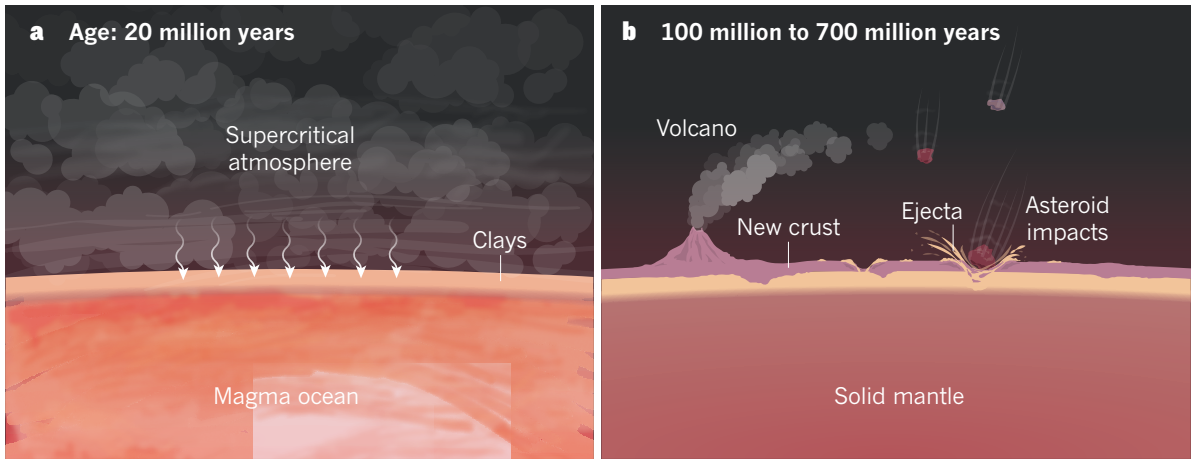

that the supercritical atmosphere would have reacted with the crust to form clays during this time (Fig. 1a).

Magma oceans exist for relatively short periods in the context of geological timescales, and so the authors' mechanism for clay formation on Mars halts long before most other proposed mechanisms would have even begun. Numerous models have been proposed for the formation of Martian clays during the later Noachian period ( 4.1 billion to 3.7 billion years ago), including: the alteration of subsurface material through reactions with groundwater ${ }^{6}$; alteration of crustal material through reactions with water at high temperatures, driven by asteroid impacts ${ }^{7}$; and surface weathering ${ }^{8}$. Cannon and colleagues' proposal does not exclude the possibility of later clay formation, but it does limit the amount of clays that could have formed during the Noachian. Notably, surface-weathering models of clay formation require warm, wet conditions throughout most of the Noachian period - which might have been conducive to life. But the authors' model is consistent with cold, dry Noachian conditions, which would have been unfavourable for life.

For primordial clays still to be present on Mars today, they must have survived the substantial reworking of the Martian crust that occurred as a result of widespread volcanism, disruptions by asteroid impacts and burial by impact ejecta (Fig. 1b). Cannon et al. performed computational simulations of the physical evolution of the primordial clay layer during this crustal reworking. The simulations' predictions of the clay content of the Martian regolith (the layer of loose materials, such as dust and
Figure 1 | A model for the formation of primordial clays on Mars. a, For about the first 20 million years of its existence, Mars went through a 'magma ocean' phase, during which all, or most, of the planet's interior was molten. The atmosphere was a supercritical fluid (a phase that is neither gas nor liquid) containing water and carbon dioxide. Cannon et al. ${ }^{3}$ show that minerals in Mars's crust could have reacted with steam in the atmosphere (white arrows) to form a layer of clays. $\mathbf{b}$, The magma ocean subsequently solidified, and the atmosphere changed to a gaseous state. During the next few hundred million years, volcanic activity would have generated a fresh layer of material on top of the primordial clays, and asteroid impacts would have churned up the upper crustal layers. c, The authors' computational simulations show that this chain of events would have resulted in the observed patterns of partially exposed clay.

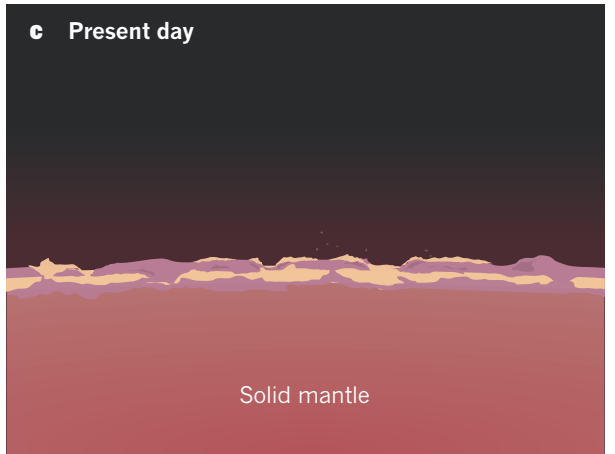


broken rock, that overlays the bedrock) and of large clay exposures at the surface are consistent with present-day observations of Mars (Fig. 1c).

According to the simulations, the primordial clay layer remains as a mostly continuous layer buried at depths of 15-25 kilometres under volcanic and impact ejecta, but exposed near impact craters. The simulations also show that the dearth of clays in the northern Martian hemisphere can be explained by the disruption of the primordial layer by the Borealis impact - the collision of a single, large body with Mars that is thought to have occurred in this region. In the southern hemisphere, the buried clay layer might correspond to a lowdensity crustal layer that has been identified by studies of the gravity and topography of Mars ${ }^{9}$.

Models of magma-ocean evolution on Earth have sometimes included crustal hydration ${ }^{10}$, but, unlike for Mars, there is no geological record for Earth that goes back more than 3.8 billion years. The primordial clays on Mars therefore provide a unique window into this hot, early stage of planet formation. For example, they will have compositions that reflect the atmospheric composition before it was altered by the loss of gases to space. By contrast, Noachian clays formed under very different conditions, and will therefore be compositionally distinct. More experimental and modelling work is needed to determine the chemical signatures of the different formation mechanisms. Measurements made by robot missions on Mars, such as NASA's Curiosity rover and the future Mars 2020 rover, might help to constrain these models.

Some of the assumptions of the primordialclay scenario will also need to be tested further. Cannon and co-workers' model assumes that the crustal porosity is initially high, allowing instantaneous alteration of the entire crustal thickness by supercritical fluid. However, clay minerals have larger volumes per unit mass than do the unaltered crustal minerals, and so the formation of clay in the upper crust will cause an expansion that might lower the porosity in this region. This could hinder clay formation at lower levels by sealing off the passages through which supercritical fluid travels to interact with the lower crust.

Finally, the clays formed in Cannon and colleagues' experiments have a different mineral structure from that of the vast majority of clays detected on Mars by remote sensing. A second stage of alteration might therefore need to be invoked to produce the structures observed on the red planet ${ }^{11}$. Further experiments must be performed in the laboratory to identify exactly which clay phases are produced, as a key step towards identifying the primordial clays on the surface of Mars.

Laura Schaefer is in the School of Earth and Space Exploration, Arizona State University, Tempe, Arizona 85287, USA.

e-mail: lkschaef@asu.edu
1. Ehlmann, B. L. et al. Space Sci. Rev. 174, 329-364 (2013).

2. Wordsworth, R. et al. Icarus 222, 1-19 (2013).

3. Cannon, K. M., Parman, S. W. \& Mustard, J. F. Nature 552, 88-91 (2017).

4. Elkins-Tanton, L. T. Earth Planet. Sci. Lett. 271, 181-191 (2008).

5. Kruijer, T. S. et al. Earth Planet. Sci. Lett. 474, 345-354 (2017).

6. Ehlmann, B. L. et al. Nature 479, 53-60 (2011).
7. Tornabene, L. L. et al. J. Geophys. Res. Planets $\mathbf{1 1 8}$ 994-1012 (2013).

8. Carter, J., Loizeau, D., Mangold, N., Poulet, F. \& Bibring, J.-P. Icarus 248, 373-382 (2015).

9. Baratoux, D. et al. J. Geophys. Res. Planets 119, 1707-1727 (2014).

10.Sleep, N. H., Zahnle, K. \& Neuhoff, P. S. Proc. Natl Acad. Sci. USA 98, 3666-3672 (2001).

11.Catalano, J. G. J. Geophys. Res. Planets $\mathbf{1 1 8 ,}$ 2124-2136 (2013).

\title{
PARKINSON'S DISEASE
}

\section{Vivid views of the PINK1 protein}

\begin{abstract}
Structures of an unusual enzymatic domain in PINK1 provide insights into how this protein regulates the function of organelles called mitochondria, and how mutations in PINK1 contribute to Parkinson's disease. SEE ARTICLE P.51
\end{abstract}

\section{SALIMA DAOU \& FRANK SICHERI}

$\mathrm{W}$ hen essential cellular organelles called mitochondria that act as the cell's energy factories are damaged, the cell's response is coordinated by two proteins - PINK1 and parkin ${ }^{1}$. Mutations in the genes that encode these proteins are among the most prevalent in hereditary early-onset Parkinson's disease $e^{2}$. Our understanding of how parkin functions and how mutations in parkin contribute to Parkinson's disease has benefited immensely from atomic-resolution snapshots of the protein in action ${ }^{3-5}$. But owing to difficulties in atomic-level imaging of PINK1, our understanding of its equally important role in these processes has been hindered. Two studies (one on page 51 by Schubert et al. ${ }^{6}$, and one in eLife by Kumar et al. ${ }^{7}$ ) have overcome these hurdles to provide near atomic-scale views of PINK1, providing invaluable insight into its mechanism of action.

PINK1 belongs to a class of enzyme called protein kinases, which change the behaviour of their target proteins by attaching a phosphate group to them (phosphorylation). When mitochondria are healthy, PINK1 levels are repressed. In response to mitochondrial stress, PINK1 migrates to the mitochondrial outer membrane, where it accumulates and selfphosphorylates to fully activate its kinase domain. Activated PINK1 phosphorylates a small protein called ubiquitin, and this phospho-ubiquitin binds to parkin, promoting the latter's ability to be phosphorylated by PINK1 on its ubiquitin-like (UBL) domain. These steps ultimately lead to the enzymatic activation of parkin - an E3 ligase enzyme that attaches ubiquitin to neighbouring proteins. Ubiquitin acts as a marker that tags proteins for degradation by other cellular machinery and so promotes the clearance of damaged mitochondria ${ }^{1}$.

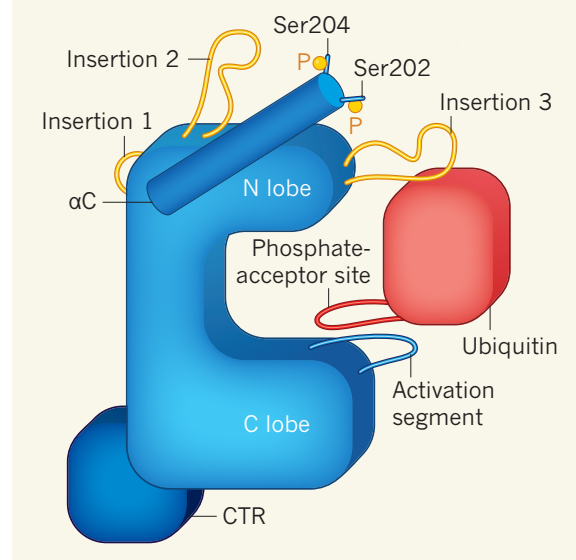

Figure 1 | Schematic of the protein kinase domain of PINK1 protein bound to ubiquitin. PINK1 is an enzyme that adds phosphate groups $(\mathrm{P})$ to itself and its substrates to modify their behaviour. PINK1 has several typical features of protein kinases - amino- and carboxy-terminal lobes ( $\mathrm{N}$ and $\mathrm{C}$ lobes, respectively), a regulatory $\mathrm{aC}$ helix and an activation segment. In addition, it has several atypical features - three insertion loops, and an unusual C-terminal region (CTR). Two groups ${ }^{6,7}$ have solved structures of PINK1, alone or bound to a mutant form of its substrate, ubiquitin. These structures revealed that insertion 2 is well positioned to influence the $\alpha \mathrm{C}$ helix and hence regulate enzyme activity. Insertion 3 provides a large contact surface that enables substrate binding. PINK1 selfphosphorylates on the amino-acid residues serine (Ser) 202 and 204 - an atypical feature that seems to promote substrate binding and catalysis by mediating the positioning of insertions 2 and 3. The phosphate-acceptor site of ubiquitin is exposed by a large conformational change, which is induced by interaction with PINK1. 